\title{
Identifying the barriers and facilitators to transforming a university hospital into a Major Trauma Centre: a qualitative case study using the Theoretical Domains Framework
}

\author{
Neil Roberts ${ }^{1 *}$, Fabiana Lorencatto ${ }^{2}$, Joanna Manson ${ }^{1}$, Jan Jansen ${ }^{3}$ \\ From London Trauma Conference 2014 \\ London, UK. 9-12 December 2014
}

\section{Background}

Scotland is currently implementing a trauma network with four Major Trauma Centres (MTCs). Transforming successful teaching hospitals in to MTCs is likely to generate various beliefs amongst staff. The Theoretical Domains Framework (TDF) is a tool to elicit and analyse beliefs [1]. This study applied the TDF to explore barriers/facilitators to MTC establishment.

\section{Method}

Semi-structured interviews were conducted with 10 participants from a single hospital prior to MTC designation including clinicians, nursing and management staff. A topic guide was designed using the TDF. Interview transcripts were analysed following a framework analysis approach and coded according to TDF domains. Themes were analysed for relevance according to prevalence, expressed importance, discordance and underlying evidence base.

\section{Results}

1728 utterances were coded into 98 themes, of which 57 were classified as relevant barriers/facilitators. Themes addressed 6 key areas: Beliefs towards becoming a MTC (e.g. My optimism/pessimism is conditional upon availability of necessary resources), resource demands (e.g. Recruitment is difficult, and may be affected by (not) becoming a MTC), current capability (e.g. Motivation for trauma varies between departments/individuals), knowledge/skill development (e.g. Maintaining skills is important, as is developing

\footnotetext{
* Correspondence: robertsneil88@gmail.com

'Barts Centre for Trauma Sciences, Blizard Institute, Queen Mary, University of London, London, UK

Full list of author information is available at the end of the article
}

them), trauma teams and a structured trauma pathway (e.g. Someone should lead/coordinate care of trauma patients), and performance improvement processes (e.g. The organisational culture is (not) supportive and geared towards performance improvement).

\section{Conclusions}

This study identified a range of barriers and facilitators likely to influence the transition of this hospital into a MTC. Findings highlight a need for clear systems, processes, communication and teamwork, and delineate the complexity of participants' motivation and optimism/ pessimism. This provides a basis for developing targeted interventions to facilitate the implementation process. This is a replicable method of evidence-based serviceimprovement, which can be applied elsewhere throughout acute care.

\footnotetext{
Authors' details

${ }^{1}$ Barts Centre for Trauma Sciences, Blizard Institute, Queen Mary, University of London, London, UK. ${ }^{2}$ Health Services Research and Management, School of Health Sciences, City University London, London, UK. ${ }^{3}$ Department of Intensive Care, Aberdeen Royal Infirmary, Aberdeen, UK.

Published: 11 September 2015

\section{Reference}

1. Cane J, et al: Validation of the theoretical domains framework for use in behaviour change and implementation research. Implement Sci 2012, 7:37.

doi:10.1186/1757-7241-23-S2-A10
Cite this article as: Roberts et al:: Identifying the barriers and facilitators
to transforming a university hospital into a Major Trauma Centre: a
qualitative case study using the Theoretical Domains Framework.
Scandinavian Journal of Trauma, Resuscitation and Emergency Medicine 2015
23(Suppl 2):A10.
}

